\title{
A NOTE ON MAXIMALLY RESOLVABLE SPACES
}

\author{
V. TZANNES \\ Department of Mathematics \\ University of Patras \\ Patras, Greece \\ (Received November 23, 1988 and in revised form June 2, 1989)
}

ABSTRACT. A.G. E1'kin [1] poses the question as to whether any uncountable cardinal number can be the dispersion character of a Hausdorff maximally resolvable space.

In this note we prove that every cardinal number $\aleph>\aleph_{1}$ can be the dispersion character of a metric (hence, maximally resolvable) connected, locally connected space. We also proved that every cardinal number $\kappa>\kappa_{0}$ can be the dispersion character of a Hausdorff (resp. Urysohn, almost regular) maximally resolvable space $X$ with the following properties: 1) Every continuous real-valued function of $X$ is constant, 2) For every point $x$ of $X$, every open neighborhood $U$ of $x$, contains an open neighborhood $V$ of $x$ such that every continuous real-valued function of $V$ is constant. Hence the space $X$ is connected and locally connected and therefore there exists a countable connected locally connected Hausdorff (resp. Urysohn or almost regular) maximally resolvable space (not satisfying the first axiom of countability).

KEY WORDS AND PHRASES. Metric, countable spaces, connected, locally connected, maximally resolvable, Urysohn, almost regular spaces.

1980 AMS SUBJECT CLASSIFICATION CODE. 54E35, 54D05, 54 G20.

\section{INTRODUCTION.}

In the sequel we denote by $\aleph_{0}$ the first infinite cardinal number and by $\aleph$ the first uncountable cardinal number.

For a topological space $X$, the dispersion character $\Delta(X)$ of $X$ is the least among the cardinals of nonvoid opens sets. The space $X$ is called maximally resolvable (Ceder [2]) if it has isolated points or $X$ is the union of $\Delta(X)$ pairwise disjoint sets, called resolvants each of which intersects each nonvoid open set in at least $\Delta(\mathrm{X})$ points.

A topological space $X$ is said to be 1) Urysohn, if for every two distinct points $x, y$ of $x$ there exist open neighborhoods $v, U$ of the points $x, y$ such that $\bar{v} \cap \bar{U}=\phi$, 
2) Regular at a point $x$, if for every open neighborhood $U$ of $x$, there exists an open neighborhood $v$ of $x$ such that $\bar{V} \subseteq U$. 3) Almost regular, if there exists a dense subset of $X$ at every point of which the space $X$ is regular.

Let $X$ be a set and let $\left\{Y^{1}: 1 \varepsilon I\right\}$ be a family of subsets of $X$ with each $Y^{1}$ having a topology. Assume that for every $(i, j) \varepsilon I \times I$, both 1) the topologies of $Y^{i}$, $\mathrm{Y}^{j}$ agree on $\mathrm{Y}^{i} \cap \mathrm{Y}^{j}$ and 2) each $\mathrm{Y}^{i} \cap \mathrm{Y}^{j}$ is open in $\mathrm{Y}^{i}$ and in $\mathrm{Y}^{j}$. Then the weak topology in $X$ induced by $\left\{Y^{1}: 1 \in I\right\}$ is $\tau=\left\{U: U \cap Y^{1}\right.$ is open in $Y^{1}$ for every $\left.1 \varepsilon I\right\}$.

Every metric space is maximally resolvable, (W. Sierpinski [3]). Every space satisfying the first axiom of countability is, also, maximally resolvable, (J.C. Ceder [2]).

2. MAIN RESULTS.

THEOREM 2.1. Every cardinal number $K>\aleph_{1}$ can be the dispersion character of a metric connected, locally connected space.

PROOF. Let $(X, d)$ be a metric connected locally connected space with dispersion character $\kappa_{1}$. We first construct a sequence of sets $x_{0}, x_{1}, \ldots x_{n}, \ldots$, then we define a metric $d^{*}$ on the set $Y=\bigcup_{n=0} X_{n}$ and we prove that $\left(Y, d^{*}\right)$ is the required space.

For an arbitrary point $x$ of $X$ we set

$$
x_{0}=\{x\} \text {. }
$$

We set $Y(x)=X \backslash\{x\}$ and we consider the set

$$
X_{1}=\{x\} \cup \quad \underset{1}{i} \in I_{1} Y^{i}(x)
$$

where $I_{1}$ is an index set such that $\left|I_{1}\right|=N$ and $Y^{1}(x)$ is the 1 -copy of $Y(x)$ attached to the point $x$. Assume also that $Y^{1}(x) \cap Y^{f}(x)=\phi$ for every $1, j \varepsilon I_{1}, 1 \neq f$. Similarly the set $X_{2}$ is defined as

$$
x_{2}=x_{0} U x \in X_{1} X_{0}\left(\{x\} U_{i \in I_{2}}^{U} Y^{1}(x)\right)
$$

where $I_{2}$ is an index set such that $\left|I_{2}\right|=x, Y(x)=X \backslash\{x\}$ and $Y^{1}(x)$ is the 1 -copy of $Y(x)$ attached $t$ the point $x$ of $X_{1} \backslash X_{0}$. Assume also that $Y^{i}(x) \cap Y^{j}(x)=\phi$ for every $1, j \in I_{1} \cup I_{2}, \quad i \neq j$ and that $Y^{i}(x) \cap Y^{j}(y)=\phi$ for every $x \neq y, \quad 1, j \varepsilon I_{1} \cup I_{2}$. Using Induction, the set $X_{n}$ is defined as

$$
x_{n}=x_{n-2} U\left(x \in x_{n-1} \mid X_{n-2}\left(\{x\} U_{1 \in I_{n}} Y^{1}(x)\right), n=3,4, \ldots\right.
$$

where $I_{n}$ is an index set such that $\left|I_{n}\right|=K, Y(X)=X \backslash\{x\}$ and $Y^{1}(X)$ is the 1-copy of $Y(x)$ attached to the point $x$ of $X_{n-1} \backslash X_{n-2^{*}}$ (It should be observed that to every point 
$x$ of $X_{n-1} \backslash X_{n-2}$ are attached $x$ pairwise disjoint copies of $\left.Y(x)\right)$. Assume also that $Y^{i}(x) \cap Y^{j}(x)=\phi \underset{n}{\text { for every } i, j \varepsilon} \underset{k=1}{U} I_{k}, 1 \neq j$ and that $Y^{i}(y) \cap Y^{j}(y)=\phi$ for every $x \neq y, i, j \in \underset{k=1}{U} I_{k}$.

We consider the set $Y=\bigcup_{n=1} X_{n}$ on which we define a metric $d^{*}$ as follows: Let $a, b$ be two arbitrary points of $Y$ and $n, m$ be the minimal integers for which

a $\varepsilon X_{n}, b \varepsilon X_{m}$. Suppose $n<m$ and let $Y^{i}\left(a_{n-1}\right), i \varepsilon I_{n}, a_{n-1} \varepsilon X_{n-1} \backslash X_{n-2}$ and $y^{j}\left(b_{m-1}\right), j \varepsilon I_{m}, b_{m-1} \varepsilon X_{m-1} \backslash X_{m-2}$ be the copies of $Y\left(a_{n-1}\right)$ and $Y\left(b_{m-1}\right)$, where the points $a, b$ belong respectively. The space $\mathrm{Y}^{j}\left(\mathrm{~b}_{\mathrm{m}-1}\right) \cup\left\{\mathrm{b}_{\mathrm{m}-1}\right\}$ is homeomorphic to the space $(X, d)$. The point $b_{m-1}$ of $X_{m-1} \mid X_{m-2}$ belongs to a copy $Y^{k}\left(b_{m-2}\right)$, $k \varepsilon I_{m-1}$, $b_{m-2} \varepsilon X_{m-2} \backslash X_{m-3}$ and the space $Y^{k}\left(b_{m-2}\right) \cup\left\{b_{m-2}\right\}$ is homeomorphic to ( $\left.x, d\right)$. Continuing in this manner we find a point $b_{m-(m-n)}=b_{n}$ belonging to $x_{n} \mid x_{n-1}$. If $a=b_{n}$ then we define

$$
d *(a, b)=d\left(b, b_{m-1}\right)+\sum_{i=1}^{m-n-1} d\left(b_{m-1}, b_{m-(i+1)}\right) .
$$

If $a \neq b_{n}$ then continuing the above process in a "parallel" way for both points $a, b_{n}$ we find a finite number of points $a_{n-1}, a_{n-2}, \ldots, a_{n-k}$ and a finite number of points $b_{n-1}, \quad b_{n-2} \cdots b_{n-k}$ such that $a_{n-k}=b_{n-k}$ for some $k, 1<k<n-1$. In this case we define

$$
d *(a, b)=d *\left(a, a_{n-k}\right)+d *\left(b, b_{n-k}\right) .
$$

It is easily verified that $d^{*}$ is a metric for the set $Y$ and that $\left(Y, d^{*}\right)$ is a connected locally connected space with dispersion character $\aleph$.

COROLLARY 2.1. Every cardinal number $k>\aleph_{0}$ can be the dispersion character of a Hausdorff (resp. Urysohn, almost regular) maximally resolvable space $Y$ with the following properties:

1) Every continuous real-valued function of $Y$ is constant.

2) For every point $a$ of $Y$, every open neighborhood $U$ of a contains an open neighborhood $V$ of a such that every continuous real-valued function of $V$ is constant.

PROOF. Let $(X, \tau)$ be a countable connected, locally connected Hausdorff (resp. Urysohn, almost regular) space (Iliadis and Tzannes [4]). We construct the set $Y$ as in Theorem 2.1 above and we consider the space $(Y, \tau)$ where $\tau$ is the weak topology induced by the (pairwise disjoint) spaces $Y^{1}(x)$, where $x \in X_{n}\left|X_{n-1}, 1 \varepsilon I_{n},\right| I_{n} \mid=\kappa$, $n=1,2, \ldots$.

It can easily be proved that $Y$ is Hausdorff (resp. Urysohn, or almost regular) having dispersion character $\kappa$.

In order to prove that $\mathrm{Y}$ is maximally resolvable we $\mathrm{fix}$ an index $\alpha \varepsilon \mathrm{I}_{\mathrm{n}}$ for every $\mathrm{n}=1,2, \ldots$, and we consider the sets 
and

$$
\begin{aligned}
& D_{1}^{\alpha}=Y^{\alpha}(x), \alpha \varepsilon I_{1} \\
& D_{2}^{\alpha}=\underbrace{Y^{\alpha}\left(x_{1}\right), \alpha \varepsilon I_{2}}_{x_{1} \varepsilon X_{1} \backslash X_{0}} \\
& \dot{D}_{n}^{\alpha}=\underbrace{}_{x_{1} \varepsilon X_{n-1} \backslash X_{n-2}} Y^{\alpha}\left(x_{1}\right), \alpha \varepsilon I_{n} \\
& D^{\alpha}=\bigcup_{n=1}^{\infty} D_{n}^{\alpha} .
\end{aligned}
$$

We set $D=\left\{D^{\alpha}: \alpha \varepsilon I_{n}, n=1,2, \ldots,\right\}$ and we observe that $D$ consists of $r$ pairwise disjoint dense sets each of which intersects every open set in points and whose union is $Y \backslash\{x\}$, hence $Y \backslash\{x\}$ is maximally resolvable and therefore $Y$ is maximally resolvable.

We now prove that every continuous real-valued function $f$ of $Y$ is constant (and hence $Y$ is connected). Let a be an arbitrary point of $Y$ and $n$ be the minimal integer for which a $\varepsilon X_{n}$. The point a belongs to a copy $Y^{1}\left(a_{n-1}\right)$, I $\varepsilon I_{n}$ attached to the point $a_{n-1}$ of $X_{n-1} \mid X_{n-2}$. The space $Y^{1}\left(a_{n-1}\right) \cup\left\{a_{n-1}\right\}$ is homeomorphic to $X$ and since $X$ is countably connected it follows that $f\left(Y^{1}\left(a_{n-1}\right) \cup\left\{a_{n-1}\right\}\right)=c$. Similarly, the point $a_{n-1}$ belongs to a copy $Y^{j}\left(a_{n-2}\right), j \varepsilon I_{n-1}$ attached to $a_{n-2}$ of $X_{n-2} \backslash X_{n-3}$ and $f\left(Y^{j}\left(a_{n-2}\right) \cup\left\{a_{n-2}\right\}\right)=c$.

It is obvious that the point $a_{n-(n-1)}$ belongs to $Y^{k}(x)$, where $k \varepsilon I_{1}$ and $\{x\}=X_{0}$ hence $f\left(Y^{k}(x) \cup\{x\}\right)=c$ and therefore $f(a)=f(x)=c$, for every point a of $Y$.

Similarly is proved property (2) (and hence $Y$ is also locally connected).

COROLLARY 2.2. There exists a countable connected locally connected Hausdorff (resp. Urysohn or almost regular) maximally resolvable space (not satisfying the first axiom of countability).

PROOF. Let $Z$ be a countable Hausdorff (or Urysohn space not satisfying the first axiom of countability. We first embed $Z$ in a countable Hausdorff (or Urysohn) almost regular space X [4, Corollary 1]. Then, we construct the space $Y$ as in Corollary 2.1 above considering $\left|I_{n}\right|=\aleph_{0}$ for every $n=1,2, \ldots$ Since $Z \subseteq X \subseteq Y$ it follows that at every point $x \in Z$ the space $Y$ does not satisfy the first axiom of countability.

\section{REFERENCES}

1. EL'KIN, A.G., Resolvable Spaces which are not Maximally Resolvable, Vestnik Moskov. Math. 24(4) (1969), 116-118.

2. CEDER, J.G., On Maximally, Resolvable Spaces, Fund. Math. 55(1) (1964), 87-93.

3. SIERPINSKI, W., Sur la decomposition des éspaces tretriques en ensembles disjoint, Fund. Math. 36 (1949), 68-71.

4. ILIADIS, S. and TZANNES, V., Spaces on which Every Continuous Map into a given Space is Constant, Can. J. Math. 36 (1986), 1281-1298. 


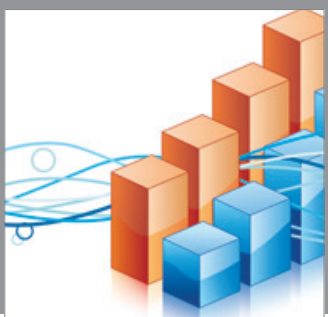

Advances in

Operations Research

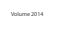

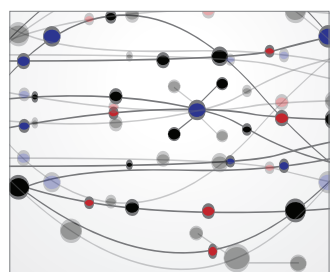

\section{The Scientific} World Journal
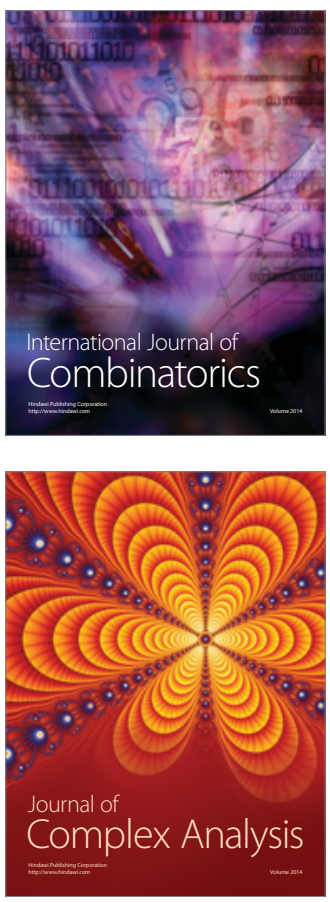

International Journal of

Mathematics and

Mathematical

Sciences
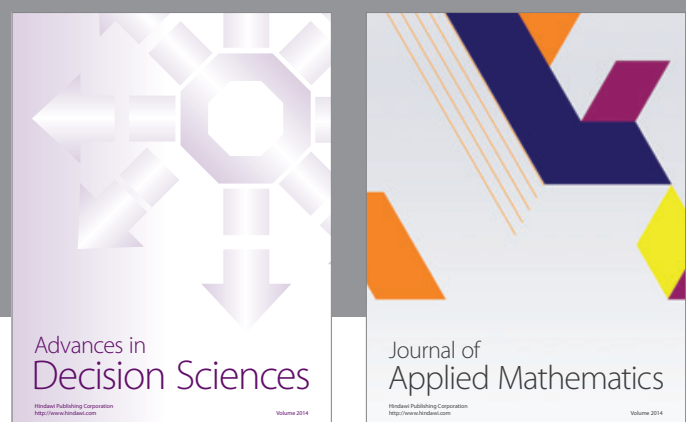

Journal of

Applied Mathematics
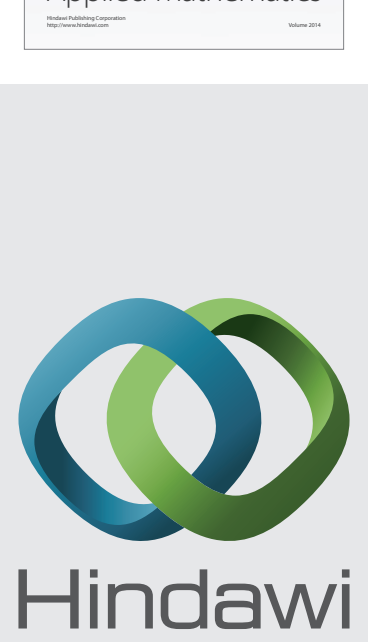

Submit your manuscripts at http://www.hindawi.com
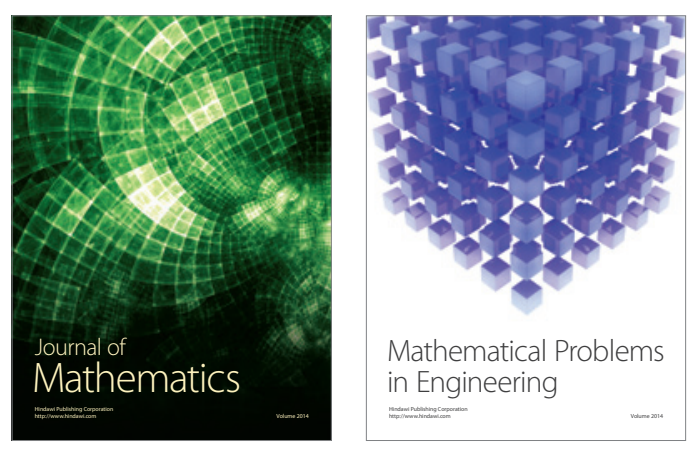

Mathematical Problems in Engineering
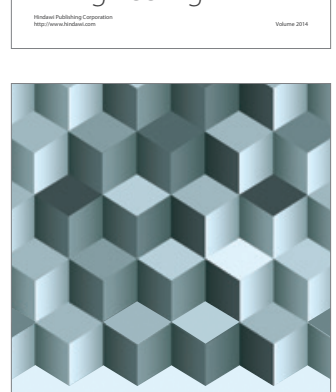

Journal of

Function Spaces
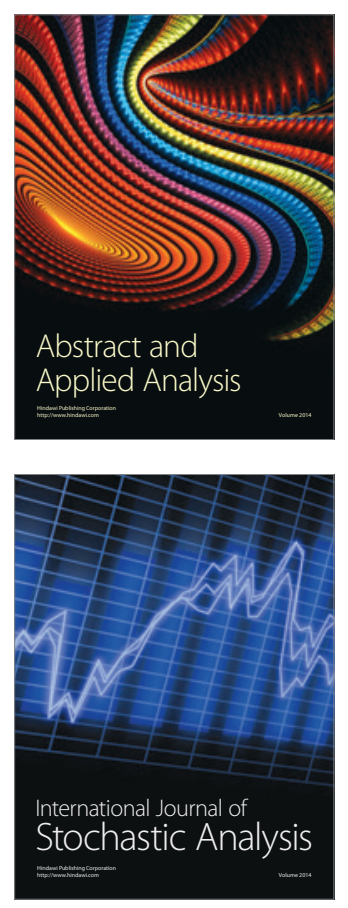

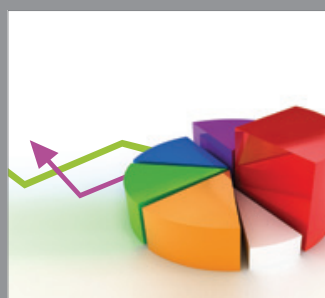

ournal of

Probability and Statistics

Promensencen
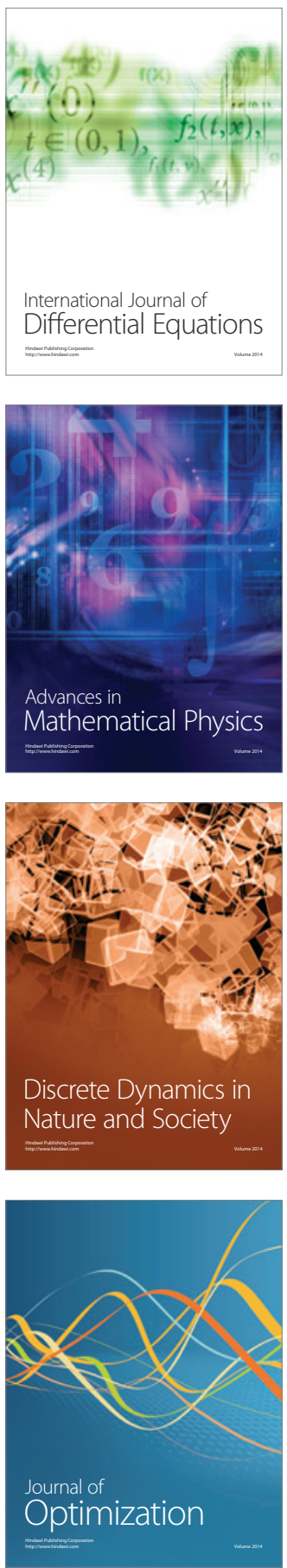